\title{
Study on Dynamic Reconfiguration of Distribution Network Considering Distribution Generation
}

\author{
Xin Geng*, Honghai Niu, Jun Chen and Bing Li \\ Research and Development Center of NR Electric Co., Ltd., Nanjing 211102, China \\ Corresponding Email: genx@nrec.com
}

\begin{abstract}
A new dynamic reconfiguration method considering distribution generation (DG) was presented. The dynamic reconfiguration problem was solved from three aspects: firstly, the time interval partition of the entire scheduling period was optimized by load distribution variation index; secondly, a plurality of time intervals were optimized and reconfigured by using the multi-objective particle swarm optimization model of multi-period encoding; finally, the optimal time interval numbers was determined by gradually approaching the falling threshold of net loss. If the system contained DG, DG's output curve was determined by the analysis of its dynamic characteristics. The results of the test showed that the proposed method was effective to solve the dynamic reconfiguration with DG.
\end{abstract}

Keywords. Dynamic reconfiguration, distribution generation, multi-objective particle swarm optimization, distribution network reconfiguration.

\section{Introduction}

Distribution Network Reconfiguration contains static reconfiguration and dynamic reconfiguration. Static reconfiguration optimizes the network at a time section, and dynamic reconfiguration optimizes the network considering the time varying which has practical significance for application. With the development of Smart Gird requiring the high penetration of Distribution Generation, the research on the network reconfiguration with DG is essential and meaningful [1].

Dynamic reconfiguration of distribution network is applied to adjust the topology structure of distribution network dynamically on the premise of the switching times constraint according to the dynamic load variation over a period, so that the optimal running state of the distribution network can be achieved. The objective function of dynamic reconfiguration is different from the static reconfiguration. Static reconfiguration only take into account the optimization target of distribution network under a certain time cross section while the objective function of dynamic reconfiguration reveals the optimization goal over a period. At present, the minimum network loss in a scheduling cycle is usually treated as the objective function which is described as:

$$
\min \int_{t_{b}}^{t_{e}} P_{l o s s}(t) \cdot \mathrm{d} t
$$


where $P_{\text {loss }}(t)$ is the network loss at time $t$. In practice, the continuous load curve of the power distribution system is usually approximately replaced with trapezoidal curve to discretize the continuous variables. The discrete objective function can be written as

$$
\min \sum_{t=1}^{T} P_{\text {loss }}(t) \cdot \Delta t
$$

where $P_{\text {loss }}(t)$ is the network loss, $t$ represents an hour, $T$ is the number of time segments. $T$ equals 24 for a scheduling cycle of a day.

Considering that the frequent network topology transformation will greatly reduce the service life of the switchgear, the overall switching times limit in the time cycle should be added in addition to the traditional static constraints (including power flow constraints, branch capacity constraints, network topology constraints)

$$
N_{\text {total }} \leq N_{\text {total } \max }
$$

where $N_{\text {total }}$ is the switching times, $N_{\text {total } \max }$ is the maximum switching times limit.

According to the mathematical model of distribution network dynamic reconfiguration, the relation and difference between dynamic and static reconfiguration can be concluded as:

The time region of dynamic reconfiguration is a scheduling cycle while the time region of static reconfiguration is only a time section or a short time segment. The dynamic reconfiguration can be approximated as the composition of static reconfiguration problem and the static reconfiguration is a particular case of reconfiguration problem.

The decision variables of dynamic reconfiguration is the switch state throughout the scheduling cycle which is described as mixed integer optimization problem of discrete time systems. However, the number of control variables is constrained by the maximum switching times limit and the dynamic reconfiguration cannot be treated as the simple combination of static reconfiguration problems.

The solution method of dynamic reconfiguration is much more complicated comparing with static reconfiguration and excessive pursuit of the global optimal solution will greatly increase the complexity of the calculation. In this research, time interval segmentation method was applied to decouple the distribution network dynamic reconfiguration problem and time then the optimal number of time interval can be determined by the relation between network loss and the time interval number. The main steps are interpreted below:

(1)The whole scheduling cycle can be reasonably divided into some time intervals according to the variation of load distribution.

(2)After the partition of scheduling cycle, utilize multi-objective particle swarm optimization to code and reconfigure the time intervals.

(3)Determination of the optimal time interval number.

\section{Principle and algorithms}

\subsection{Timer interval partition of scheduling cycle [2]}

In some cases, load of various points might exhibit the same trend of variation during a period and the value change of a specific load point will not change the load distribution of the whole power distribution system. It is essential to standardize the load state at any time by 


$$
D_{i}(t)=\frac{L_{i}(t)}{L_{\text {total }}(t)}
$$

where $L_{i}(t)$ is the load of point $i$ at time $t, L_{\text {total }}(t)$ is the total load at time $t, D_{i}(t)$ is the standardized load value of point $i$ at time $t$ which represent the load proportion of point $i$ at time $t$. After the standardization of all the points, the load variation of a single point can be calculated as

$$
\Delta D_{i}\left(t_{1}, t_{2}\right)=\left|D_{i}\left(t_{1}\right)-D_{i}\left(t_{2}\right)\right|
$$

where $D_{i}\left(t_{1}, t_{2}\right)$ represents the load variation of point $i$ from time $t_{1}$ to time $t_{2}$, then the total variation of load distribution from time $t_{1}$ to time $t_{2}, \Delta D\left(t_{1}, t_{2}\right)$ can be calculated as

$$
\Delta D\left(t_{1}, t_{2}\right)=\sum_{i=1}^{m}\left|D_{i}\left(t_{1}\right)-D_{i}\left(t_{2}\right)\right|
$$

A high $\Delta D\left(t_{1}, t_{2}\right)$ value represents large variation of load distribution from time $t_{1}$ to time $t_{2}$. The standardized value is obviously reasonable for describing the change of load distribution and the load change will not be submerged by the variation of total load. In addition, a threshold of variation of load distribution $\Delta D_{\max }$ is needed for the time interval partition.

Throughout the time interval partition, the threshold $\Delta D_{\max }$ is first set as the initial value $\Delta D_{\max , 0}$, then the scheduling cycle is divided according to $\Delta D_{\max }$. Appropriate $\Delta D_{\max , 0}$ will greatly reduce iterations of the algorithm, which is related to the presupposed time interval number $n$. The initial value can be calculated $\Delta D_{\max , 0}$ from the largest variation of load distribution $\max \Delta D$ during a scheduling cycle:

$$
\Delta D_{\max , 0}=\frac{\max \Delta D}{n}
$$

If the time interval number doesn't equal the presupposed value $n, \Delta D_{\max }$ will be adjusted to divide the scheduling cycle again until the time interval number equals $n$. Regarding the time interval number after $i$ steps of iteration $n_{i}$, the threshold of variation of load distribution for the next step of iteration $\Delta D_{\text {max }, i+1}$ can be adjusted as:

$$
\Delta D_{\max , i+1}= \begin{cases}\Delta D_{\max , i}+\varepsilon, & n_{i}>n \\ \Delta D_{\max , i}-\varepsilon, & n_{i}<n\end{cases}
$$

The time interval partition process is illustrated in Figure 1.

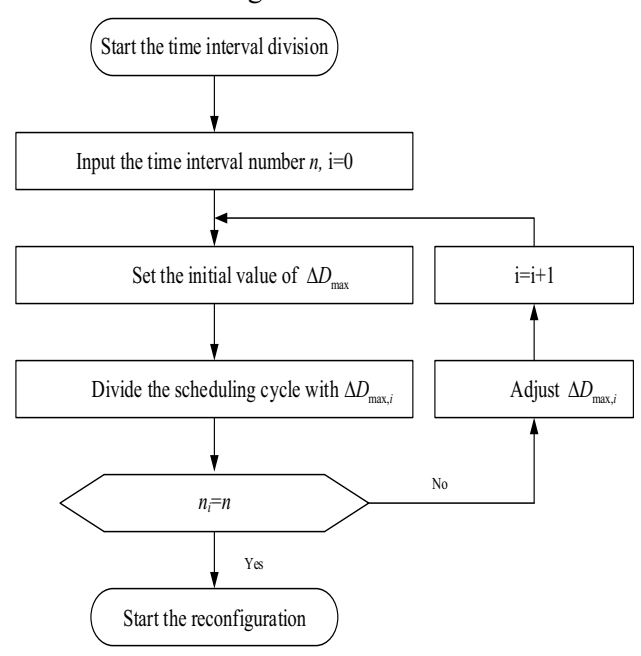

Fig. 1. The process of timer interval partition. 


\subsection{Dynamic reconfiguration of distribution network [3]}

The mathematical model of dynamic reconfiguration requires the minimum network loss in a scheduling cycle and meets the maximum switching times constraint conditions. After the partition of scheduling cycle, the fitness function can be modified as:

(1)The minimal total network loss

$$
\min f_{1}=\sum_{i=1}^{n} P_{\text {loss }}(i) \cdot \Delta t(i)
$$

where $P_{\text {loss }}(i) 、 \Delta t(i)$ is the total network loss and segment length of time interval $i$, respectively.

(2)The minimal switching times

$$
\min f_{2}=N_{\text {total }}
$$

where $N_{\text {total }}$ represent the total switching times.

Regarding the multi-objective particle swarm optimization [4] (MOPSO) method, there are $n$ substrings for the encoding of $n$ time intervals and every substring represents the encoding form of the network topology. Figure 2 illustrates the encoding form for 3 timer intervals.

$$
\left[\begin{array}{ccccc}
x_{1,1} & x_{1,2} & x_{1,3} & x_{1,4} & x_{1,5} \\
x_{2,1} & x_{2,2} & x_{2,3} & x_{2,4} & x_{2,5} \\
x_{3,1} & x_{3,2} & x_{3,3} & x_{3,4} & x_{3,5}
\end{array}\right] \begin{aligned}
& \text { Substring 1 } \\
& \text { Substring 2 } \\
& \text { Substring 3 }
\end{aligned}
$$

Fig. 2. The encoding form for 3 timer intervals

Different time interval number will result in different dynamic reconfiguration strategies, however, the reconfiguration of every time interval is a good result and the network structure will not change a lot after one step of dynamic reconfiguration. Considering the local searching ability of multi-objective particle swarm optimization, the satisfactory result will be easily obtained near the particles if the optimal solution set and the particle optimal solution 'pbest' of the previous dynamic reconfiguration step are selected as the initial group of the next dynamic reconfiguration step. The method is interpreted below:

(1)The optimal solution set of the previous dynamic reconfiguration step and substrings of the 'pbest' are extracted and put into a feasible solution library, every substring represents a feasibly good network topology structure.

(2)According to the requirements of the current dynamic reconfiguration, substrings were randomly selected from the feasible solution library to make up the particle encoding form, and the initial particle group of the current dynamic reconfiguration will be built.

Among all the feasible strategies ( $N_{\text {total }} \leq N_{\text {total } \max }$ ), the optimal one exhibit the least network loss which is the desired reconfiguration strategy.

It can be concluded from experience that more reconfiguration number within a scheduling cycle brings about more reduction network loss. However, the network structure will not change a lot with reconfiguration number increases and the economic efficiency will reduce. In addition, more reconfiguration number means more switching times and the cost of switch operation, besides the network loss, should be taken into account. In practice, it is hard to precisely determine the maintenance and operating costs of switchgear. In order to find the optimal time interval number, the following criterion is introduced:

$$
P_{\text {loss }}^{n}-P_{\text {loss }}^{n+1} \leq \varepsilon_{\text {loss }}
$$


where $n$ is the time interval number, $P_{\text {loss }}^{n}$ is the minimum network loss of $n$ time intervals considering the switching times limit, $\varepsilon_{\text {loss }}$ is the network loss reduction threshold when change the time interval number from $n$ to $n+1$. The threshold $\varepsilon_{\text {loss }}$ can be calculated as $1 \% \sim 2 \%$ of the initial network loss according to experience. Equation (11) indicates that the time interval number will be increased from $n$ to $n+1$ only if the minimum network loss reduction is beyond the threshold $\varepsilon_{\text {loss }}$. The process of dynamic reconfiguration is illustrated in Figure 3.

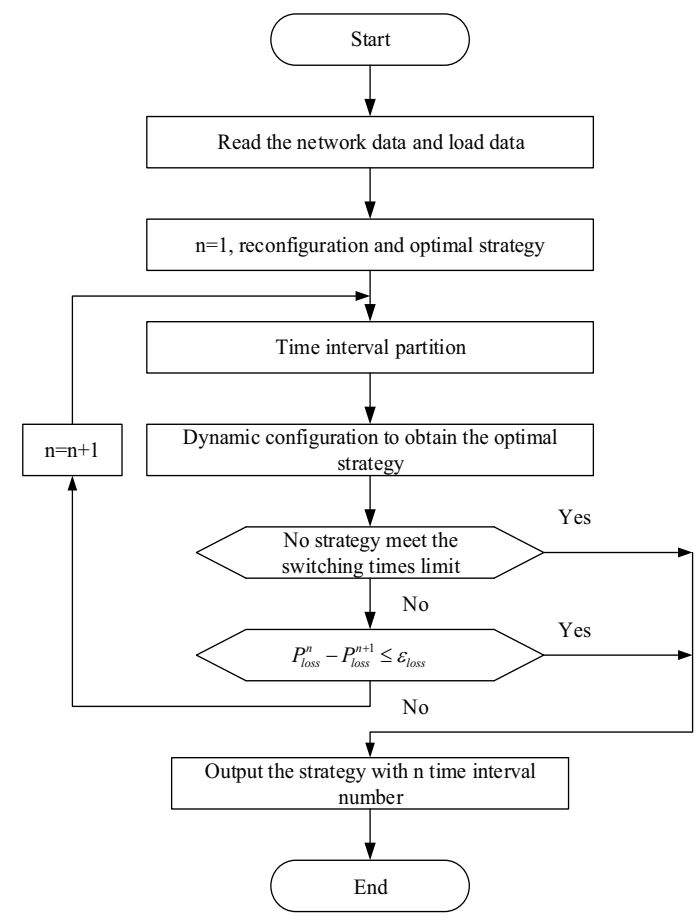

Fig. 3. The process of dynamic reconfiguration.

\section{Examples and analysis}

\subsection{Example data}

The measuring equipment is usually installed on the feeder in the substation exit, the total load variation of a feeder can be obtained and the load curve of each point is unavailable. The load curve estimation of each load point can be carried out according to the total load of the feeder and the historical data of the power station [5]. There are several types of load including resident load, industrial and commercial load.

It is assumed that resident, industrial and commercial load points are randomly distributed in the 69-node system and the standard node loads of the system is set as load peak value of each point. The sunlight intensity and wind speed data are collected from the one month historical data of a photovoltaic power station and wind power station, respectively.

For the sake of illustrating the influence of distribution generation on the distribution network dynamic reconfiguration, two different cases are discussed:

Case1: No distribution generation, only the three types of load (resident, industrial and commercial) are concerned.

Case2: Three photovoltaic power stations are connected to each of node 9,60 and 69 , three wind power stations are connected to each of node 18,48 and 27. 


\subsection{Simulation results}

Case1: No distribution generation, only the three types of load (resident, industrial and commercial) are concerned.

It is assumed that the maximum switching times $N_{\text {total } \max }$ equals 10 , the network loss reduction threshold $\varepsilon_{\text {loss }}$ is $15 \mathrm{kWh}$. The dynamic configuration results according to the variation of load distribution and the value change of total load are shown in Table 1. The time interval partition based on the variation of load distribution is 0:00 6:00, 6:00 17:00, 17:00 24:00 while the partition result based on the value change of total load is 0:00 6:00, 6:00 22:00, 22:00 24:00. Dynamic configurations of the two kinds of time interval partition are carried out for comparison. According to the time interval partition based on the variation of load distribution, the total network loss caused by the dynamic configuration is $972.59 \mathrm{kWh}$ while the time interval partition based on the value change of total load creates $983.9 \mathrm{kWh}$ of dynamic configuration network loss. It can be concluded that the proposed variation of load distribution index provides a better description of the distribution network load change then contributes to a more reasonable time interval partition in comparison with the traditional index (value change of total load).

Table 1. The results of the two dynamic configuration method.

\begin{tabular}{|c|c|c|c|}
\hline \multirow{2}{*}{$\begin{array}{c}\text { Time } \\
\text { interval } \\
\text { partition }\end{array}$} & \multicolumn{2}{|c|}{ Dynamic configuration strategy } & \multirow{2}{*}{$\begin{array}{r}\text { Net- } \\
\text { work } \\
\text { loss } \\
\text { kWh }\end{array}$} \\
\hline & $\begin{array}{c}\text { Time } \\
\text { interval }\end{array}$ & Open switching & \\
\hline \multirow{3}{*}{$\begin{array}{c}\text { Variation } \\
\text { of load } \\
\text { distribution }\end{array}$} & $0: 00 \sim 6: 00$ & $13-20,12-13,46-47,11-66,50-51$ & 62.09 \\
\hline & $6: 00 \sim 17: 00$ & $13-20,14-15,44-45,11-66,50-51$ & 759.07 \\
\hline & $17: 00 \sim 24: 00$ & $13-20,12-13,44-45,11-66,50-51$ & 151.26 \\
\hline Total & & 6 switching actions & 972.6 \\
\hline \multirow{3}{*}{$\begin{array}{l}\text { Value } \\
\text { change of } \\
\text { total load }\end{array}$} & $0: 00 \sim 6: 00$ & $13-20,12-13,46-47,11-66,50-51$ & 2.09 \\
\hline & $6: 00 \sim 22: 00$ & $13-14,12-13,45-46,11-66,52-53$ & 893.30 \\
\hline & $22: 00 \sim 24: 00$ & $13-20,12-13,47-48,11-66,50-51$ & 28.03 \\
\hline Total & & 10 switching actions & 983.4 \\
\hline
\end{tabular}

Table 2 shows the network loss, loss reduction rate and switching times throughout the dynamic configuration of a scheduling cycle without switching times constraint considering different time interval numbers. When the time interval number increases from 1 to 3 , the network loss falls sharply and the switching times are not less than 10. When the time interval number is beyond 4 , the network loss of the system decreases slowly while the switching times increases sharply. It is suitable to divide one day into 2 4 time intervals [6].

Table 2. The results of dynamic configuration considering different time interval numbers.

\begin{tabular}{|c|c|c|c|}
\hline $\begin{array}{c}\text { Time interval } \\
\text { number }\end{array}$ & $\begin{array}{c}\text { Network loss after } \\
\text { configuration } \\
(\mathrm{kWh})\end{array}$ & $\begin{array}{c}\text { Loss reduction } \\
\text { rate } \\
(\%)\end{array}$ & Switching times \\
\hline 1 & 1125.7 & 47.84 & 0 \\
\hline 2 & 988.55 & 54.20 & 4 \\
\hline
\end{tabular}




\begin{tabular}{|c|c|c|c|}
\hline 3 & 972.59 & 54.93 & 8 \\
\hline 4 & 970.41 & 55.03 & 12 \\
\hline 5 & 969.56 & 55.07 & 16 \\
\hline 6 & 968.34 & 55.13 & 22 \\
\hline 7 & 965.92 & 55.25 & 24 \\
\hline 8 & 964.71 & 55.30 & 26 \\
\hline 9 & 963.34 & 55.36 & 28 \\
\hline 10 & 963.34 & 55.36 & 28 \\
\hline
\end{tabular}

Case 2: Three photovoltaic power stations are connected to each of node 9, 60 and 69, three wind power stations are connected to each of node 18,48 and 27.

The maximum switching times $N_{\text {total } \max }$ is set as 10 and the network loss reduction threshold $\varepsilon_{\text {loss }}$ is $15 \mathrm{kWh}$. The results of the proposed dynamic configuration method are shown in Table 3.

Table 3. The dynamic configuration results of case 2 .

\begin{tabular}{|l|l|l|}
\hline \multicolumn{2}{|l|}{ Dynamic configuration strategy } & Network loss \\
\hline Time intervals & Open switching & kWh \\
\hline $0: 00 \sim 14: 00$ & $13-20,13-14,8-9,9-10,50-51$ & 515.01 \\
\hline $14: 00 \sim 24: 00$ & $13-20,12-13,47-48,11-66,50-51$ & 250.12 \\
\hline Total & 6 switching actions & 778.13 \\
\hline
\end{tabular}

The scheduling cycle is divided into two time intervals: 0:00 14:00, 14:00 24:00. The dynamic configuration results of case 2 is totally different from the results of case 1 which is attributed to the change of node load curve caused by the distributed generators. The network loss reduction is not very large regarding 3 time intervals and the unnecessary switching actions can be avoided if the cycle is divided into 2 intervals. Table 3 indicates that the proposed method provides effective strategies for the dynamic configuration of distribution network considering distribution generation [7].

\section{Conclusions}

The time interval partition method based on the variation of load distribution index is proposed. Multi-period encoding is applied to deal with the coordination among the switching states of different time intervals and the strategies for the dynamic configuration of distribution network is provided considering distribution generation. The simulation algorithms is carried out on the IEEE 69-node system sample and the proposed variation of load distribution index provides a more reasonable time interval partition in comparison with the traditional index (value change of total load). According to the dynamic configuration results considering different time interval numbers, the real economic value contributed by the increase of time interval number is limited.

\section{References}

1. Thomas A, Goran A, Lennart S. Distributed Generation: a Definitio. Electric Power System Research, 2001, $57(3): 195.204$.

2. Lin W M, Cheng F S, Tsay M T. Distribution feeder reconfiguration with refined genetic algorithm. IEE Proceedings of Generation, Transmission and Distribution, 2000,147 (6):349-354. 
3. Jonathan E Fieldsend, Sameer Singh. A multi -objective algorithm based upon particle swarm optimization: An efficient data structure and turbulence. Proc of the 2002 UK Workshop on Computational Intelligence. Manchester, $2002: 37-44$.

4. Coello C A C, Pulido G T, Lechuga M S. Handling multiple objectives with particle swarm optimization. IEEE Transactions on Evolutionary Computation, 2004,8 (3):256-279.

5. Su C L. Probabilistic load-flow computation using point estimate method. IEEE Transactions on Power Systems, 2005,20(4):1843-1851.

6. Hong H P. An efficient point estimate method for probabilistic analysis. Reliability Engineering \&amp; System Safety, 1998,59(3):261-267.

7. Charytoniuk W, Chen M S, Kotas P, et al. Demand forecasting in power distribution systems using nonparametric probability density estimation. IEEE Transactions on Power Systems, 1999, 14(4):1200-1206. 